LAWRENCE LIVERMORE N A T IO N A L LABORATORY

\title{
An Experimental Study of Drag Reduction Devices for a Trailer Underbody and Base
}

J. M. Ortega, K. Salari

June 4, 2004

34th AIAA Fluid Dynamics Conference and Exhibit Portland, OR, United States

June 28, 2004 through July 1, 2004 
This document was prepared as an account of work sponsored by an agency of the United States Government. Neither the United States Government nor the University of California nor any of their employees, makes any warranty, express or implied, or assumes any legal liability or responsibility for the accuracy, completeness, or usefulness of any information, apparatus, product, or process disclosed, or represents that its use would not infringe privately owned rights. Reference herein to any specific commercial product, process, or service by trade name, trademark, manufacturer, or otherwise, does not necessarily constitute or imply its endorsement, recommendation, or favoring by the United States Government or the University of California. The views and opinions of authors expressed herein do not necessarily state or reflect those of the United States Government or the University of California, and shall not be used for advertising or product endorsement purposes. 


\title{
An Experimental Study of Drag Reduction Devices for a Trailer Underbody and Base
}

\author{
Jason M. Ortega ${ }^{*}$ and Kambiz Salari ${ }^{\dagger}$ \\ Lawrence Livermore National Laboratory, Livermore, CA, 94551
}

\begin{abstract}
Low speed wind tunnel measurements are made on a $1 / 16^{\text {th }}$ scale generic tractor-trailer model at a width-based Reynolds number of 325,000 . The model is fixed to a turntable, allowing the yaw angle to be varied between $\pm 14^{\circ}$ in $2^{\circ}$ increments. Various add-on drag reduction devices are mounted to the model underbody and base. The wind-averaged drag coefficient at $65 \mathrm{mph}$ is computed for each configuration, allowing the effectiveness of the add-on devices to be assessed. The most effective add-on drag reduction device for the trailer underbody is a wedge-shaped skirt, which reduces the wind-averaged drag coefficient by $2.0 \%$. For the trailer base, the most effective add-on drag reduction device is a set of curved base flaps having a radius of curvature of 0.91 times the trailer width. These curved base flaps reduce the wind-averaged drag coefficient by $18.8 \%$, providing the greatest drag reduction of any of the devices tested. When the wedge-shaped skirt and curved base flaps are used in conjunction with one another, the wind-averaged drag coefficient is reduced by $20 \%$.
\end{abstract}

\section{Nomenclature}

$A=$ cross-sectional area of the full-scale heavy vehicle

$A_{b} \quad=$ cross-sectional area of the model

$D \quad=$ aerodynamic drag

$D_{w a} \quad=$ wind-averaged drag

$C_{d} \quad=$ drag coefficient

$C_{d w a}=$ wind-averaged drag coefficient

$l_{a} \quad=$ length of the angled base flaps

$M \quad=$ weight for the numerical integration of the wind-averaged drag coefficient

$p \quad=$ probability distribution of the crosswind velocity

$R \quad=$ radius of curvature of curved base flaps

$R e_{w} \quad=$ width-based Reynolds number

$V \quad=$ wind velocity relative to the vehicle or model

$V_{t} \quad=$ vehicle velocity relative to the road

$V_{w} \quad=$ crosswind velocity

$w \quad=$ model width

$\alpha \quad=$ deflection angle of the base flaps

$\phi \quad=$ angle of the crosswind velocity relative to the vehicle velocity

$\Delta \mu \quad=$ fuel savings of gallons per mile

$v \quad=$ kinematic viscosity of air

$\rho \quad=$ air density

${ }^{*}$ Engineer, New Technologies Engineering Division, P.O. Box 808, L-644, and Member.
${ }^{\dagger}$ Engineer, New Technologies Engineering Division, P.O. Box 808, L-644, and Associate Fellow.

1

American Institute of Aeronautics and Astronautics

The work has been authored by a contractor of the U.S. Government under Contract No. W-7405-ENG-48 between the United States Department of Energy and The Regents of the University of California for the operation of LLNL. Accordingly, the U.S. Government retains a non-exclusive royalty-free license to publish or reproduce the published form of the Contribution, or allow others to do so for U.S. Government purposes. UCRL-CONF-204489 


\section{Introduction}

$\mathrm{H}$ EAVY vehicles play a vital role in the national economy by transporting throughout the country over $85 \%$ of the total value of goods in the United States. Over the past three decades, this transportation mode has been increasingly utilized, accounting for nearly all of the increases in the U.S. highway transportation energy usage. ${ }^{1}$ Unfortunately, this mode of transportation is not $100 \%$ fuel efficient. In fact, approximately $65 \%$ of the engine's power production goes into overcoming aerodynamic drag at highway speeds. These aerodynamic losses in the operation of heavy vehicles were recognized three decades ago and a considerable research effort was made to improve the aerodynamic performance of heavy vehicles.

Recently, Cooper ${ }^{2}$ provided a thorough historical overview of this effort, a summary of which is given here. As Cooper describes, the results of this research led to the development of first- and second-generation aerodynamic drag reduction devices. The first-generation devices focused upon cab-mounted air deflectors, fairings, side extenders, and front-end rounding, which resulted in relative decreases in the drag coefficient of approximately 15$31 \%$. These aerodynamic improvements were accepted by the heavy vehicle industry because they required modification only to the tractors, which were fewer in number than the trailers. Additionally, the tractor owners could recover their investment from the fuel cost savings. The second-generation devices consisted of base flaps, trailer skirts, and tractor/trailer gap sealers. The base flaps are made of several flat plates that are attached to the trailer base and are angled slightly inward. These plates cause the freestream flow to turn more sharply inward into the trailer wake, resulting in a reduction of the drag coefficient. The trailer skirts are flat plates that extend beneath the trailer bottom and span the distance between the rear tractor wheels and the trailer wheels. These skirts function by shielding the trailer wheels and axles from the crosswinds, thereby streamlining the trailer wheels/axles assembly. Some trailer skirt designs also include additional skirts that are located behind the trailer wheels. The tractor/trailer gap sealer is made of a flexible curtain that extends from the trailer front to the tractor rear. The sealer operates by preventing a cross flow from forming in the gap between the tractor and trailer when the heavy vehicle experience crosswinds. Eliminating this cross flow and the resulting momentum exchange to the heavy vehicle results in a reduction of the drag coefficient. Each of these three devices results in approximately a 3-15\% reduction of the aerodynamic drag. Despite their effectiveness, these second-generation devices were not accepted on a widescale basis in the heavy vehicle industry. The reason for this was that these devices required modification to the trailers, which were more in number than the tractors, thus requiring a greater initial investment. Additionally, the base flaps required manual deployment and retraction, which often interfered with loading operations, the side skirts tended to restrict access to the trailer underside, and the tractor/trailer gap sealers did not have the durability to withstand years of use without frequent replacement. However, with rising fuel costs and the need to reduce the U.S.'s dependence on foreign oil, there is a greater motivation today to decrease the fuel usage of heavy vehicles. In 1996, the U.S. Department of Energy founded the Office of Heavy Vehicle Technologies (OHVT) with the purpose of researching and developing concepts that could increase the energy efficiency of heavy vehicles and reduce vehicle emissions. ${ }^{3}$ One of the specific milestones of the OHVT is to develop the technologies that will allow Class 7 and 8 heavy vehicles to achieve a fuel economy of $10 \mathrm{mpg}$ at $65 \mathrm{mph}$ by 2004 . Consequently, the secondgeneration devices, which were previously unaccepted, may become the means by which this goal can be achieved. Yet, to gain the acceptance of the heavy vehicle industry, the second-generation devices must be developed in a manner that not only maximizes the aerodynamic drag reduction, but also provides designs that are practical for normal operations.

In an effort to accomplish this goal of improving the fuel efficiency of heavy vehicles, the present study investigates further optimization of the base flaps and alternate forms of trailer skirt designs. Previous research ${ }^{2,4}$ on the angled base flaps has demonstrated that this concept is capable of reducing the drag by as much as $10 \%$ when the flaps are deployed at equal angular deflections. However, it is quite possible that an optimum configuration may be one in which the top and side base flaps have slightly different angular deflections. In the subsequent sections, we explore this possibility. Additionally, we investigate the drag reducing capability of curved base flaps, which have previously shown to perform about as well as straight base flaps. ${ }^{4}$ To circumvent the shortcomings of straight side skirts, which have been shown to reduce the drag by as much as $15 \%$, we investigate three variations of a wedge trailer skirt concept that may provide the drag reduction benefits of straight side skirts, while still allowing access to the trailer underside. The layout of the remainder of the paper is as follows. In Section 2, a description of the experimental setup and procedures is presented. The wind-averaged drag coefficient, which is a useful quantity for comparing the effectiveness of various drag reduction devices, is described in Section 3. The performance of the 
base flaps, trailer skirts, and their combinations are discussed in Section 4. The summary and conclusions are presented in Section 5.

\section{Experimental Setup and Procedures}

The effectiveness of trailer underbody and base drag reduction devices is assessed by making axial force measurements on a $1 / 16^{\text {th }}$ scale generic tractor-trailer model (Fig. 1), which is a simple representation of a nearfuture tractor/trailer. ${ }^{5}$ The wind tunnel measurements are made in the NASA Ames Fluid Mechanics Laboratory open-circuit wind tunnel, which has a contraction ratio of 9:1, a test section size of $813 \mathrm{~mm} \times 1219 \mathrm{~mm}$, and freestream turbulence level of $0.15 \%$. The wind tunnel is operated at a nominal dynamic pressure and tunnel speed of $574 \mathrm{~N} / \mathrm{m}^{2}$ and $103 \mathrm{~m} / \mathrm{s}$, respectively, both of which are measured from a pressure ring upstream of the tunnel test section. The model measures $162 \mathrm{~mm} \times 225 \mathrm{~mm}$ x $1238 \mathrm{~m}$, giving a nominal width-based Reynolds number of $R e_{w}=V w / v=325,000$, where $w$ is the model width $=162 \mathrm{~mm}$, and $v$ is the kinematic viscosity of air. Since this

$\mathrm{Re}_{\mathrm{w}}$ is about an order of magnitude less than that of a heavy vehicle operating at highway conditions, care is taken to ensure that the flow is completely turbulent over the model by attaching boundary layer trips made of $1 \mathrm{~mm}$ thick serrated tape to the tractor nose. The effectiveness of these boundary layer trips was confirmed in a Reynolds number sensitivity test, which showed that the change in drag coefficient with Reynolds number is of order $10^{-7}$ for tunnel speeds ranging from $60-140 \%$ of the nominal value used in this study. The percentage of blockage of the model in the tunnel test section is $3 \%$ at $0^{\circ}$ yaw. Since this data may be used in the future for CFD code validation, no corrections are made to the force data to account for the model blockage. The model is composed of two pieces: a $324 \mathrm{~mm}$ long tractor constructed of Balsa Foam and a $914 \mathrm{~mm}$ long trailer constructed from $6 \mathrm{~mm}$ and $10 \mathrm{~mm}$ thick acrylic sheets. The entire model is covered with flat black paint for enhanced contrast in oil surface flow visualization studies (not shown). An array of holes are machined onto the trailer base, sides, and top for use in mounting various add-on drag reduction devices. When not in use, these holes are covered over with tape to prevent any airflow through them. The wheels of the model are made from ten $38 \mathrm{~mm}$ radius half-cylinders that are mounted on the underside of the tractor and trailer. The two front wheels have width of $20 \mathrm{~mm}$, while the other eight wheels have a width of $51 \mathrm{~mm}$.

The model is mounted on a six-component balance (Northrop MK XVA 0.75 ") at a height that places the model wheels $25 \mathrm{~mm}$ above the tunnel floor. The force balance is capable of measuring loads of $133.4 \mathrm{~N}$ in the axial direction. All of the axial force measurements presented below are made with respect to the body-axis coordinate system shown in Fig.1. For a typical experimental run, the axial force loads the balance to approximately $11 \%$ of the balance capacity. The measurement uncertainty of the calculated drag coefficients is \pm 0.008 , which accounts for both the balance accuracy and measurement repeatability. The signals from the force balance are digitized and stored on a computer using BDAS 9.2 data acquisition software. ${ }^{6}$ For each data point collected during an experimental run, the force balance signals are sampled for 8 seconds ( 200 model lengths) at $250 \mathrm{~Hz}$. Doubling the sampling time to 16 seconds resulted in a $1 \%$ change in the time-averaged force and moment measurements. The force balance is mounted on a sting that has two vertical $13 \mathrm{~mm}$ diameter threaded rods that attach the entire model/balance assembly to a $851 \mathrm{~mm}$ diameter turntable. The turntable is driven by a computer-controlled servo motor (Parker Compumotor), which can rotate the model to within $\pm 0.1^{\circ}$. For each model configuration, the force measurements are made at yaw angles, $\psi$, ranging from $\pm 14^{\circ}$ in $2^{\circ}$ increments. To assess the repeatability of the measurements, as well as hysteresis in the flow patterns over the model, the data are acquired for both increasing and decreasing yaw angle directions. Subsequent measurements demonstrate that any hysteresis effects are negligible.

Four skirt designs (Fig. 2) are tested on the trailer underside: a long wedge skirt (Fig. 2a) which has an apex angle of $10^{\circ}$; a short wedge skirt (Fig. 2b) which has an apex angle of $22^{\circ}$; a short wedge skirt with an upstream center skirt (Fig. 2c); and two conventional straight side skirts (Fig. 2d), which are used as a reference for making performance comparisons with the other three skirt designs. Each of the trailer skirts are constructed from $2 \mathrm{~mm}$ thick aluminum sheet metal that is folded to form a right angle and is then cut to a width that places the 

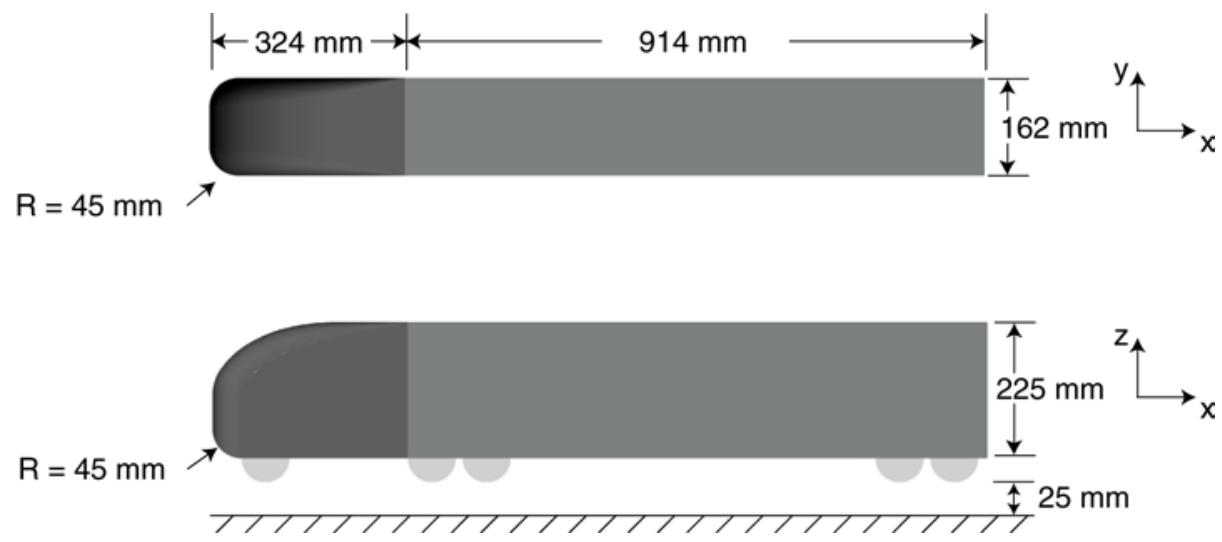

Figure1. Model of the cab-over-engine tractor-trailer used in the wind tunnel study.
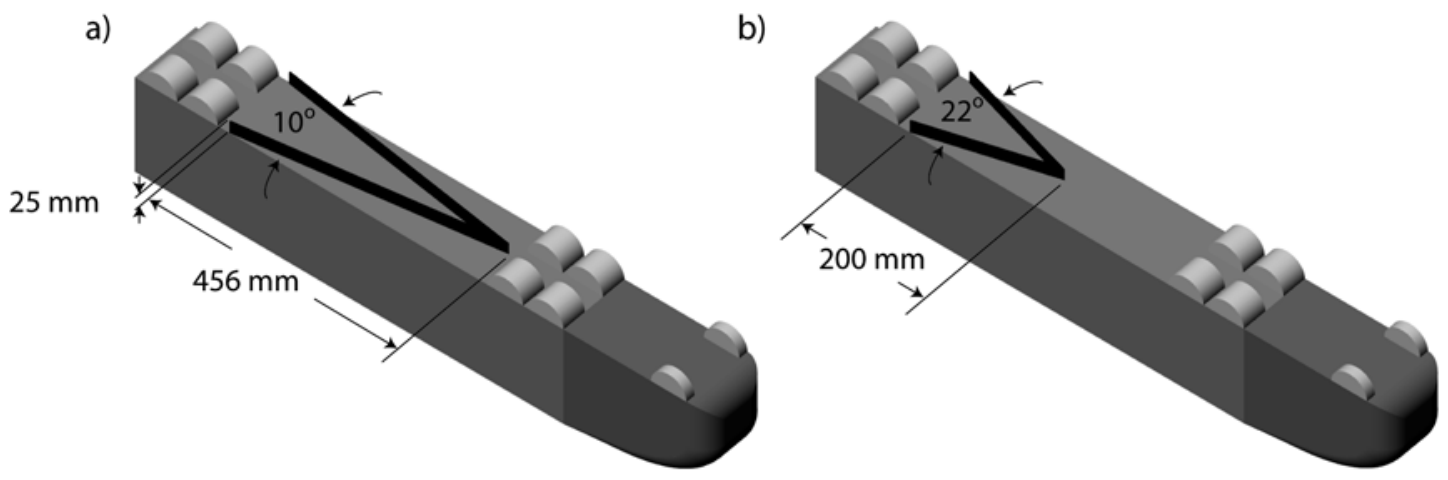

c)

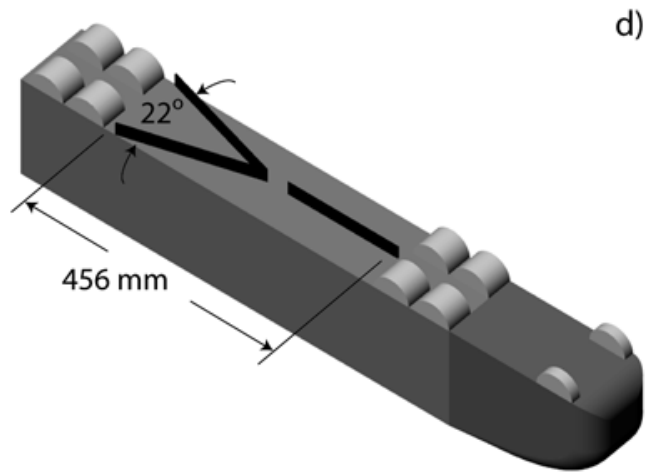

d)

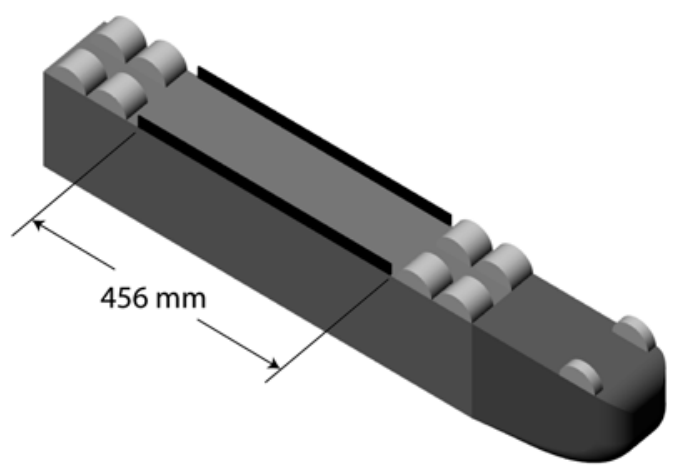

Figure 2. Skirt designs used to reduce the trailer underbody aerodynamic drag: a) long wedge skirt; b) short wedge skirt; c) short wedge skirt with center skirt; d) straight side skirts. (Note that the gap between the center skirt and short wedge skirt in c) is to allow clearance for the sting mount.)

bottom of the skirt $25 \mathrm{~mm}$ beneath the trailer underside. Each of the skirts is fastened to the trailer underside by means of several machine screws.

A set of angled flaps is tested as a means of reducing the base drag of the model (Fig. 3a). The four flaps are constructed from $2 \mathrm{~mm}$ thick aluminum piano hinges that extend a distance of $l_{a}=56 \mathrm{~mm}$ from the trailer base when 
placed at a deflection angle, $\alpha$, of $0^{\circ}$. Non-dimensionalizing $l_{a}$ by $\sqrt{A_{b}}$ gives a value of 0.291 , where $A_{b}$ is the cross-sectional area of the model. Cooper ${ }^{4}$ showed this value of $l / \sqrt{A_{b}}$ to be greater than the minimum length necessary to achieve the full drag reduction benefit of the base flaps. During the experiments, the top and side flaps are independently deflected to $5^{\circ}, 10^{\circ}, 15^{\circ}$, and $20^{\circ}$, while the bottom flap is maintained at an angle of $0^{\circ}$. The corners of the base flaps are sealed with tape for each deflection angle, yielding an octagonal-shaped cavity on the base of the trailer. The four curved base flap devices are constructed using a rapid prototyping technique (selective laser sintering), which forms a single-pieced design. The curved base flaps extend a distance of $48 \mathrm{~mm}$ from the trailer base and have radii of curvature, $R$, of $52,79,148$, and $288 \mathrm{~mm}$. In units of the trailer width, the radii of curvature are $0.32,0.49,0.91$, and 1.78 .

a)

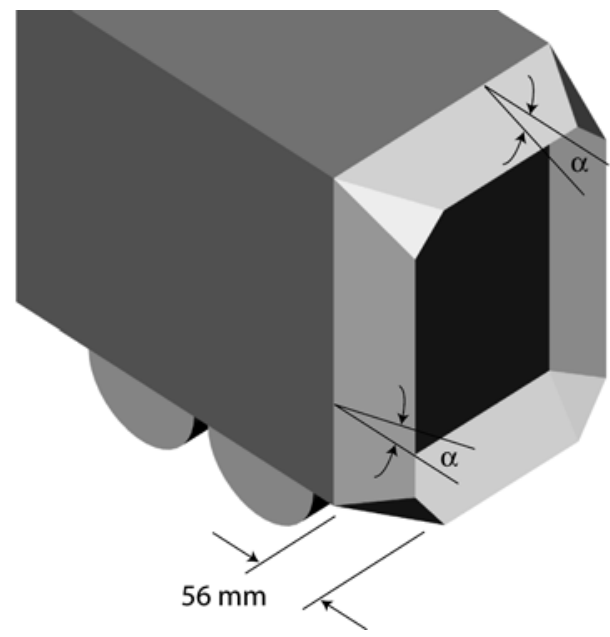

b)

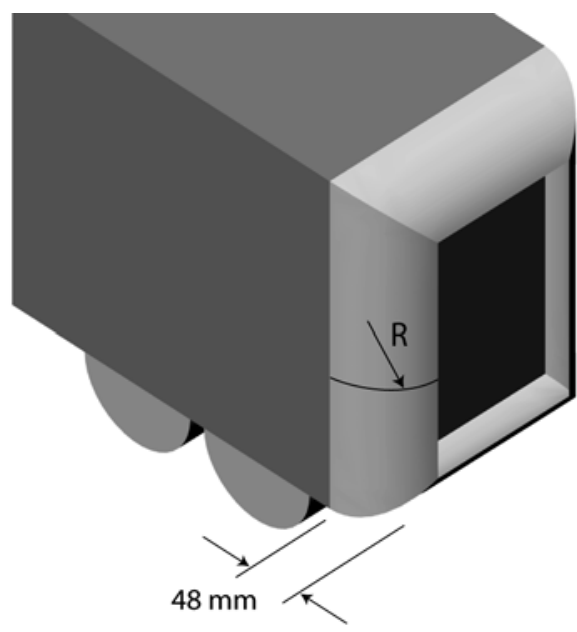

Figure 3. Base flap designs: a) angled base flaps; b) curved base flaps.

\section{Wind-averaged Drag Coefficient}

During the experimental runs, the drag, $D(\psi)$, on the model is measured at each yaw angle and used to compute the drag coefficient, $C_{d}(\psi)=D(\psi) /\left(1 / 2 \rho V^{2} A_{b}\right)$, where $\rho$ is the air density. While the information obtained from $C_{d}(\psi)$ is useful, it is somewhat cumbersome since the drag coefficient distribution does not summarize the performance of, say, a drag reduction device into a single quantity that can be compared with that of other devices. Taking the mean of the $C_{d}(\psi)$ is also insufficient since it does not account for the fact that the crosswind velocities cause a vehicle traveling at a particular speed to experience certain yaw angles more than others. A quantity that resolves both of these issues is the wind-averaged drag coefficient, $C_{d w a}$, which Ingram ${ }^{7}$ derived as follows. Assume that the vehicle (Fig. 4) is traveling at a velocity $V_{t}$ with respect to the roadway and there is a crosswind that has a velocity $V_{w}$ acting at an angle $\phi$ with respect to $V_{t}$. The total velocity of the wind relative to the vehicle is given by

$$
V=V_{t} \sqrt{1+2\left(V_{w} / V_{t}\right) \cos \phi+\left(V_{w} / V_{t}\right)^{2}}
$$

and the yaw angle by

$$
\tan \psi=\frac{\left(V_{w} / V_{t}\right) \sin \phi}{1+\left(V_{w} / V_{t}\right) \cos \phi}
$$




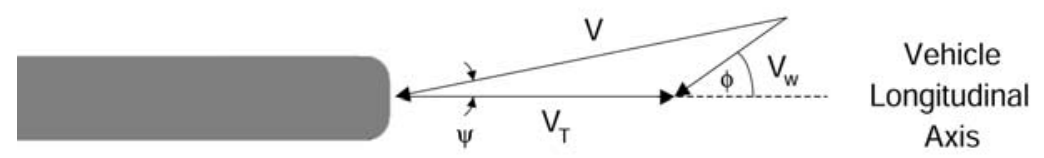

Figure 4. Schematic of the relative wind vectors.

The wind-averaged drag is found from

$$
\begin{aligned}
& D_{w a}=\int_{0}^{2 \pi} \int_{0}^{V_{w_{\max }}} D(\psi) p\left(V_{w}, \phi\right) d V_{w} d \phi \\
&=\int_{0}^{2 \pi} \int_{0}^{V_{w \max }} C_{d}(\psi) 1 / 2 \rho V^{2} A_{b} p\left(V_{w}, \phi\right) d V_{w} d \phi \\
&=\int_{0}^{2 \pi} \int_{0}^{V_{w_{\max }}} C_{d}(\psi) 1 / 2 \rho V_{t}^{2}\left[1+2\left(V_{w} / V_{t}\right) \cos \phi+\right. \\
&\left.\left(V_{w} / V_{t}\right)^{2}\right] A_{b} p\left(V_{w}, \phi\right) d V_{w} d \phi
\end{aligned}
$$

where $p\left(V_{w}, \phi\right)$ is the probability of a wind speed $V_{w}$ blowing at an angle $\phi$ with respect to the vehicle and $V_{w}$ max is the maximum wind speed. Dividing Eq. (3) by $1 / 2 \rho V_{t}^{2} A_{b}$ gives the wind-averaged drag coefficient. Assuming that the wind speed, $V_{w}$, is given by a constant national average and that it has an equal probability of occurring from any direction, such that $p\left(V_{w}, \phi\right)=1 / 2 \pi$, and that the vehicle is symmetric about its longitudinal axis, gives a windaveraged drag coefficient of

$$
C_{d w a}=\frac{1}{\pi} \int_{0}^{\pi} C_{d}(\psi)\left(1+2\left(V_{w} / V_{t}\right) \cos \phi+\left(V_{w} / V_{t}\right)^{2}\right) d \phi
$$

Equation (4) can be numerically integrated using the first-order accurate composite midpoint rule from

$$
C_{d w a}=\frac{1}{6} \sum_{j=1}^{6} M(j) C_{d}(\psi(j))
$$

where

$$
\begin{gathered}
M(j)=1+2\left(V_{w} / V_{t}\right) \cos \phi(j)+\left(V_{w} / V_{t}\right)^{2} \\
\phi(j)=\frac{\pi}{6} j-\frac{\pi}{12} \\
\psi(j)=\tan ^{-1} \frac{\left(V_{w} / V_{t}\right) \sin \phi(j)}{1+\left(V_{w} / V_{t}\right) \cos \phi(j)}
\end{gathered}
$$


Figure 5 shows a plot of $\psi(j)$ versus $M(j)$ for $V_{w}=7 \mathrm{mph}$, and $V_{t}=30,55,65$, and $75 \mathrm{mph}$. It can be seen that the smaller $V_{w} / V_{t}$, the smaller the yaw angles that are used in computing $C_{d w a}$. This demonstrates the importance of reducing the drag coefficient not only at $\psi=0$, but also over range of yaw angles that the vehicle will experience under typical operation. The drag coefficient data in the subsequent experimental measurements is fairly symmetric about $\psi=0$, such that the drag coefficients at each value of $\pm \psi$ are averaged and then used in the calculation of $C_{d w a}$. The values of $C_{d}(\psi(\phi))$ are computed by linearly interpolating the drag coefficient data from this averaged distribution to each value of $\psi(\phi(j))$. For the subsequent portion of the paper, all of the wind-averaged drag coefficients are reported for $V_{w}=7 \mathrm{mph}$ and $V_{t}=65 \mathrm{mph}$.

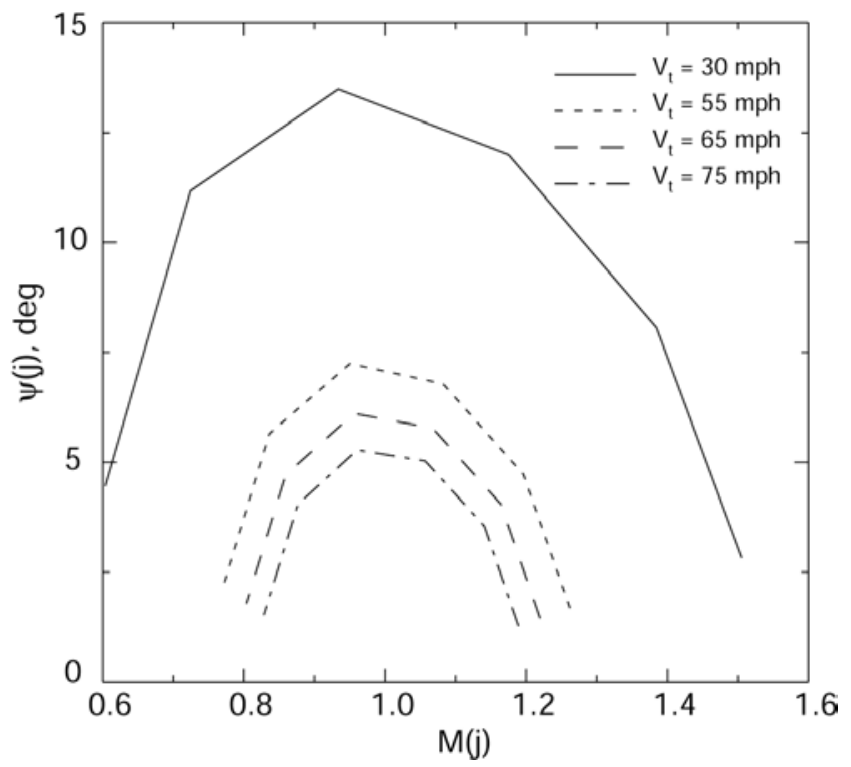

Figure 5. Yaw angles, $\psi(j)$, used in computing the wind-averaged drag coefficient (Eq. (5)) for $V_{w}=7 \mathrm{mph}$ and $V_{t}=30,55,65$, and $75 \mathrm{mph}$.

\section{Results}

In addition to the runs made with the trailer skirts and base flaps add-on devices, several baseline configuration (model with tractor and trailer wheels) cases are repeated throughout the experimental test sequence to ascertain the repeatability of the axial force measurements. Before discussing the drag reduction properties of the trailer skirts and base flaps, we consider the results of the baseline configuration.

\section{A. Baseline Configuration}

Figure 6 shows the variation of the drag coefficient with yaw angle for the baseline model geometry with and without wheels. The uncertainty in the drag coefficient measurements is \pm 0.008 , as shown pictorially in Fig. 6 . The four black bars on the horizontal axes denote the range of angles $\left(1.44^{\circ}<|\psi|<6.11^{\circ}\right)$ over which the wind-averaged drag coefficient is computed. At $\psi=0^{\circ}$, the baseline configuration without wheels has a minimum drag coefficient of $0.334 \pm 0.008$. Testing a similar model at comparable $R e_{w}$, Hammache et al. ${ }^{5}$ showed the drag coefficient of the model at $\psi=0^{\circ}$ to be 0.399 , the difference of which may be due to the fact that Hammache et al. had boundary layer 
suction upstream of the model. As $|\psi|$ is increased, the drag coefficient increases, an effect which is likely due to the additional drag caused by flow separation off the top, bottom, and downwind side of the trailer. Salari and McWherter-Payne ${ }^{8}$ performed three-dimensional numerical simulations on a similar tractor/trailer geometry and showed that such flow separation forms multiple streamwise vortices along the length of the trailer. At $\psi= \pm 10^{\circ}$, the drag coefficient has a maximum value of 0.385 , which represents a $15 \%$ increase over the value at $\psi=0^{\circ}$. The experimental study of Storms et al. ${ }^{9}$ on a similar model at higher $R e_{w}$ showed a similar relative increase in the drag coefficient at $\psi=10^{\circ}$. The wind-averaged drag coefficient for this configuration, as well as configuration with wheels, is shown in Table 1. The addition of the tractor and trailer wheels further raises the values of the drag coefficient curves and wind-averaged drag coefficients, as shown in Fig. 6 and Table 1.

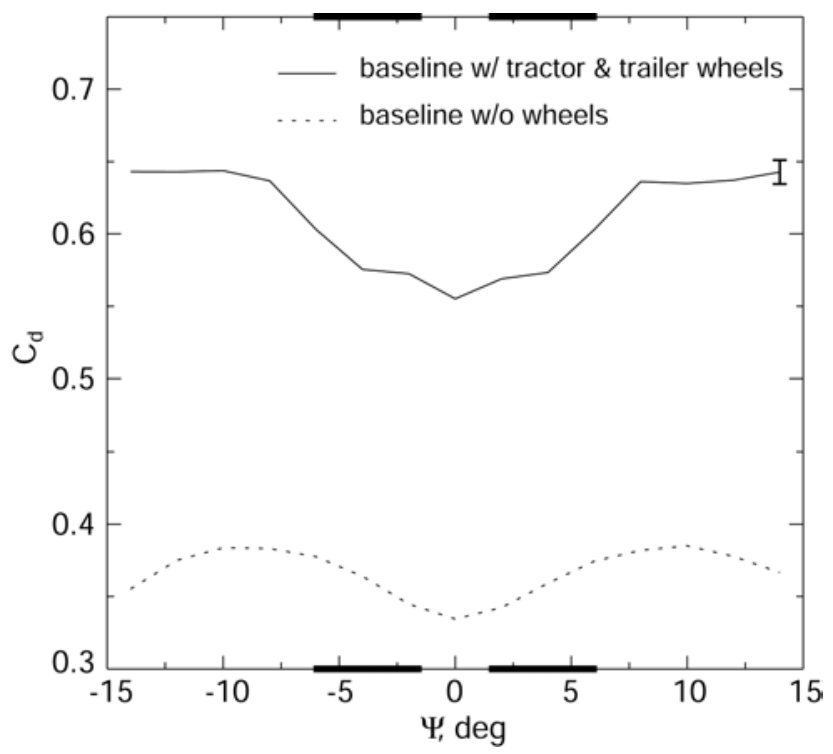

Figure 6. Drag coefficient curves for the baseline model with and without wheels. The vertical bar on the right is indicative of the measurement uncertainty, $\mathbf{\pm 0 . 0 0 8}$, of the drag coefficient. The four thick bars on the horizontal axes denote the range of yaw angles over which the wind-averaged drag coefficient is computed.

\section{B. Trailer Skirt Configurations}

Figure 7 shows the variation of the drag coefficient with yaw angle for the four trailer skirts. The drag coefficient curve corresponding to the baseline configuration is also plotted in Fig. 7 for reference. The shaded regions denote the yaw angles over which the skirts provide a reduction in the drag coefficient over that of the baseline configuration. It can be seen in Fig. $7 \mathrm{a}$ that the straight side skirts reduce the drag coefficient to a greater extent the more $|\psi|$ is increased and that they provide minimal reduction in the drag coefficient at $\psi=0^{\circ}$. A similar trend was shown by Cooper ${ }^{2}$ for both full- and model-scale tests of straight side skirts. It can be seen that the range of yaw angles over which the straight side skirts reduce the drag coefficient coincides only with a portion of the range of yaw angles that are used in computing the wind-averaged drag coefficient. This reemphasizes the importance of designing a drag reduction device that not only reduces the drag on a heavy vehicle, but also does so over the range of yaw angles that the vehicle will experience under operating conditions. The drag coefficient curve for the long wedge skirt is shown in Fig. 7b. One immediate observation that can be made is the reduction in the drag coefficient that the long wedge skirt yields at $\psi=0^{\circ}$, a behavior which is quite different than that of the straight side skirts. One possible explanation for this result is that the long wedge skirt acts to streamline the incoming flow around the rear trailer wheels at $\psi=0^{\circ}$, thereby reducing the overall drag coefficient. At larger values of $|\psi|$, the long wedge skirt reduces the drag coefficient in a manner similar to that of the straight side skirts, though to a somewhat lesser extent. The drag coefficient curves for the short wedge skirt and the short wedge skirt with the 
center skirt (Figs. 7c-d) are nearly identical, indicating that the addition of the center skirt yields no benefit in reducing the aerodynamic drag. For small yaw angles, both of these skirts provide a reduction in the drag coefficient in a manner similar to that of the long wedge skirt. However, for larger values of $|\psi|$, the short wedge skirts demonstrate minimal reduction in the drag coefficient over that of the baseline configuration. A noteworthy trend should be highlighted from the drag reduction performance of the four skirts. The straight side skirts provide the greatest drag reduction at yaw angles ranging from $4^{\circ}<|\psi|<12^{\circ}$, while yielding little benefit for smaller yaw angles. On the other hand, the short wedge skirts reduce the drag only for small yaw angles. Interestingly enough, the long wedge skirt combines the drag reduction benefits of both the straight side skirts and the short wedge skirts by reducing the drag coefficient over both yaw angle ranges.

The wind-averaged drag coefficients for the four trailer skirts are listed in Table 1. It should be noted that the trailer skirts Cooper ${ }^{2}$ tested yielded reductions in the wind-averaged drag coefficient that are much larger than those shown presently. The reason for this difference may be due to the fact that the models Cooper used had complete axles and wheels on the trailer, which would likely contribute a much greater portion to the overall vehicle drag than simple half-cylinder wheels. Hence, the installation of the skirts on the more realistic models would result in a greater drag reduction. Both short wedge skirts provide negligible reduction of the wind-averaged drag coefficient. On the other hand, it can be seen that the long wedge skirt provides the greatest drag reduction of the four trailer skirt designs. This suggests that the long wedge skirt is a design that can improve upon that of the traditional straight side skirts by not only yielding a greater reduction in the wind-averaged drag coefficient, but also allowing easier access to the trailer underside. However, additional testing of the long wedge skirt with more a realistic trailer underbody and a moving ground plane is needed before a definite conclusion can be drawn.

\section{Trailer Base Flaps}

The angled base flaps on the sides and top of the trailer are tested at independent angular deflections of $5^{\circ}, 10^{\circ}$, $15^{\circ}$, and $20^{\circ}$, while the bottom flap is maintained at a deflection angle of $0^{\circ}$. A contour plot of the wind-averaged drag coefficients for these 16 configurations is shown in Fig. 8a. One immediate observation that can be made is that the wind-averaged drag coefficient is more sensitive to changes in side angle of deflection, $\alpha_{\text {side }}$, than to changes in the top angle of deflection, $\alpha_{t o p}$. This is likely due to the fact that the side flaps have nearly twice the surface area of the top flap and, thereby, influence a larger portion of the flow field. Cooper ${ }^{4}$ also observed a greater sensitivity of the drag coefficient to the presence of the side flaps than to the top flap. It is also evident in Fig. 8a that there is a combination of $\alpha_{\text {side }}$ and $\alpha_{\text {top }}$ that yields a minimum in the wind-averaged drag coefficient in the vicinity of $\alpha_{\text {side }} \approx$ $10^{\circ}$ and $\alpha_{t o p} \approx 10^{\circ}$. The wind-averaged drag coefficients in the range of $5^{\circ} \leq \alpha_{\text {side }} \leq 15^{\circ}, 5^{\circ} \leq \alpha_{t o p} \leq 15^{\circ}$ are fit with a second-order polynomial surface to estimate the angular deflections that yield a minimum value of the windaveraged drag coefficient. Doing so gives a minimum in the polynomial surface at $\alpha_{\text {side }}=10.1^{\circ}$ and $\alpha_{\text {top }}=11.1^{\circ}$, at which location the wind-averaged drag coefficient is $0.493 \pm 0.004$. This value is $16.3 \pm 0.8 \%$ less than that of the baseline configuration. Cooper ${ }^{4}$ tested a similar set of base flaps on two heavy vehicle models and demonstrated that at an optimum flap deflection angle was $15^{\circ}$, the base flaps reduce the drag by $4-7 \%$, which is smaller than that for the base flaps in the present study. The difference between these two results may be due to the fact that the vehicle forebody of the model used in the present study is more streamlined than that in Cooper's study. As suggested by Cooper ${ }^{4}$, vehicles with a streamlined forebody shape may have increased base drag because of the higher entrainment due to thinner separating shear layers. Consequently, the base flaps on a vehicle with a streamlined forebody may reduce a greater portion of the overall vehicle drag. Fig. $8 \mathrm{~b}$ shows a plot of the drag coefficient as a function of yaw angle for a combination of flap deflection angles $\left(\alpha_{\text {side }}=\alpha_{\text {top }}=10^{\circ}\right)$ that is about equal to that which gives a minimum wind-averaged drag coefficient. It can be seen that the base flaps provide a nearly constant offset from the drag coefficient curve of the baseline configuration for all of the yaw angles tested.

As a comparison to the base flaps made of straight plates, four curved base flap configurations are tested. Fig. 8c shows the wind-averaged drag coefficients for the curved base flaps as a function of the dimensionless flap radius of curvature. To estimate the radius of curvature that gives a minimum in the wind-averaged drag coefficient, the data are fit with a second-order polynomial. The minimum value of the fitted polynomial is $0.476 \pm 0.004$, which occurs at $R / w=1.01$. This optimum configuration has a wind-averaged drag coefficient that is $19.3 \pm 0.8 \%$ less than that of the baseline configuration. This optimum reduction in the drag coefficient is the largest for any single add-on device tested in this study. In Cooper's ${ }^{4}$ investigation of curved base plates, the greatest reduction in the wind-averaged drag coefficient occurs for values of $R / w$ ranging from 0.4 to 0.5 , which are about one-half of that in the present study. This difference may again be due to the fact that the vehicle forebody of the model used in the present study 

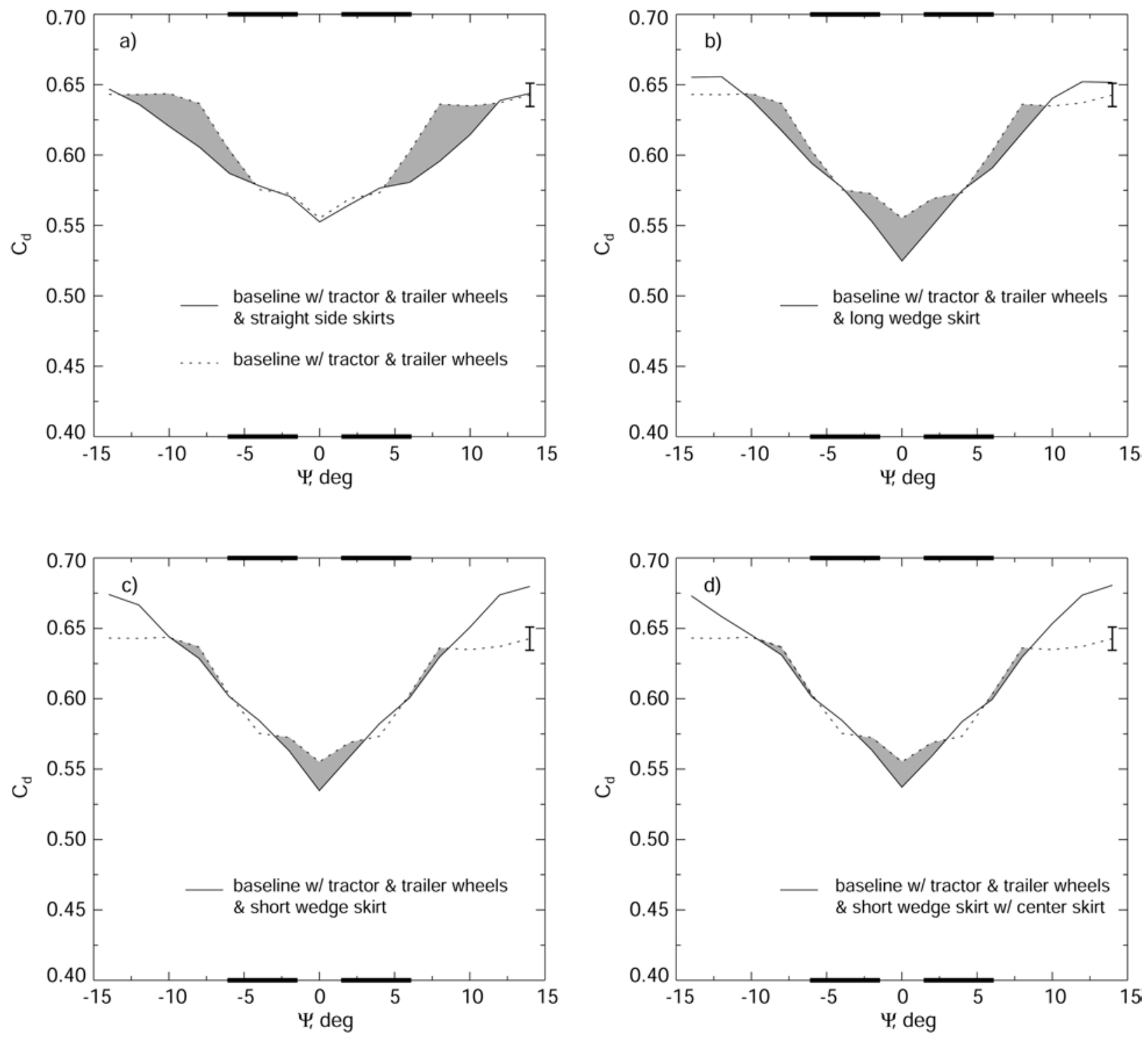

Figure 7. Drag coefficient curves for the baseline configuration with the trailer skirt devices: a) straight side skirts; b) long wedge skirt; c) short wedge skirt; d) short wedge skirt with center skirt. The shaded regions denote the yaw angles over which the skirt device results in a drag reduction. 

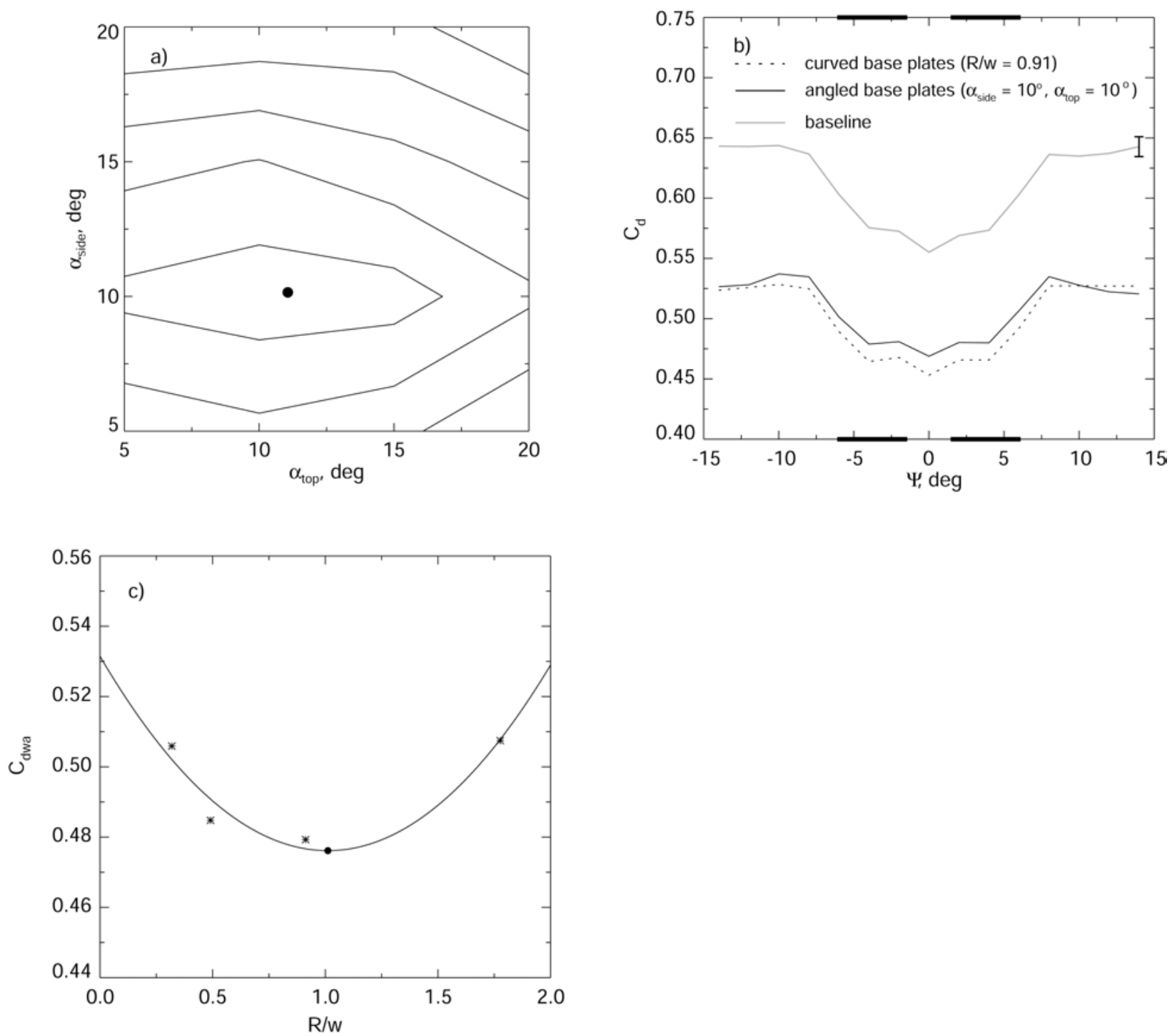

Figure 8. a) Contour plot of the wind-averaged drag coefficient of the model with angled base flaps as a function of the top, $\alpha_{\text {top }}$, and side, $\alpha_{\text {side }}$, flap deflection angles. The black symbol at $\alpha_{\text {side }}=10.1^{\circ}$ and $\alpha_{\text {top }}=11.1^{\circ}$ is the minimum wind-averaged drag coefficient $(0.493 \pm 0.004)$ found by fitting the data with a second-order polynomial surface; b) drag coefficient curves for the model configuration with angled base flaps with $\alpha_{\text {side }}=$ $10^{\circ}$ and $\alpha_{t o p}=10^{\circ}$ and for the model configuration with the curved base flaps with $R / w=0.91 ;$ c) windaveraged drag coefficient for the model with the curved base flaps as a function of the dimensionless flap radius of curvature. The black symbol at $R / w=1.01$ is the minimum wind-averaged drag coefficient $(0.476 \pm 0.004)$ found by fitting the data with a second-order polynomial.

is more streamlined than that in Cooper. ${ }^{4}$ Fig. $8 \mathrm{~b}$ shows the drag coefficient curve as a function of the yaw angle for a set of curved base flaps that have a radius of curvature $(R / w=0.91)$ of the same order as that of the optimum configuration. In a manner similar to that of the straight base flaps, the curved base flaps give a fairly constant reduction in the drag coefficient over the range yaw angles tested. The measured wind-averaged drag coefficients for the angled and curved base flap configurations closest to the optimal flap deflection angle and radius of curvature, respectively, are listed in Table 1. 


\section{Combination of Trailer Skirts and Base Flaps}

Having analyzed the performance of the trailer skirts and base flaps on an individual basis, we now assess the effectiveness of combinations of these devices and determine the configuration that results in the greatest drag reduction. Figure 10 shows the percent reduction in the wind-averaged drag coefficient relative to the baseline case for combinations of trailer skirts, base flaps, and, for reference, the individual devices. The uncertainty in these measurements is $\pm 0.8 \%$ and is denoted by the error bar in the plot. It can be seen that of all the devices tested, the combination of the curved base flaps $(R / w=0.91)$ with the long wedge skirt gives the greatest reduction in the windaveraged drag coefficient. This combination results in a $20.0 \pm 0.8 \%$ reduction in the wind-averaged drag coefficient, the majority of which is due to the contribution of the curved base flaps. Figure 11 shows a plot of the drag coefficient as a function of yaw angle for this combination of devices and, for comparison, the combination of the angled base flaps with the long wedge skirt. The wind-averaged drag coefficients for these two configurations are shown in Table 1. Note that the variation of the drag coefficient with yaw angle is reminiscent of that of the long wedge skirt, where the drag coefficient has a distinct minimum at $\psi=0^{\circ}$. A comparison of the wind-averaged drag coefficients of the model with trailer skirts and the model with base flaps indicates that the base flaps provide a substantially larger drag reduction than that of the skirts. Assuming that the trends in the wind-averaged drag coefficients are applicable to full-sale heavy vehicles, these results could have important implications for the current day trucking industry. Clearly, if a trucking fleet decided to purchase a single add-on drag device to obtain the greatest drag reduction, the choice of curved base flaps would be the best alternative. However, the angled base flaps may be more attractive from an investment point of view since their rather simple design would be much less costly to manufacture and, thereby, require a smaller initial investment.

Before concluding this section, a useful exercise is to estimate the annual fuel savings that can be achieved by using the best-case configuration of the curved base flaps $(R / w=0.91)$ and the long wedge skirt on an actual heavy vehicle. It should be kept in mind that this estimate is somewhat approximate given the low Reynolds number of these measurements and the fact that no blockage corrections have been applied to the drag coefficient data. Cooper ${ }^{2}$ conducted a similar fuel savings exercise and we follow his analysis below. The formula for the fuel savings per mile is given by

$$
\Delta \mu=4.37 \times 10^{-7} V_{T}^{2} \Delta C_{d w a} A
$$

where $\Delta C_{d w a}$ is the decrease in the wind-averaged drag coefficient, $V_{t}$ has the units of mph, and $A$ is the frontal area $\left(\mathrm{ft}^{2}\right)$ of the vehicle. In Eq. (6), it has been assumed that the fuel consumption of the vehicle is $0.38 \mathrm{lb} / \mathrm{hp}$-hr. For the optimum configuration, $\Delta \mu=0.0219$ gallons per mile, where $V_{t}=65 \mathrm{mph}, A=100.6 \mathrm{ft}^{2}$ ( $16 \times$ the model area), and $\Delta C_{d w a}=0.118$. This value of $\Delta \mu$ can be converted to dollar per truck per year by multiplying it by the 1997 average annual miles traveled per truck $(48,095 \mathrm{mi})^{10}$ and a conservative value of $\$ 1.50$ per gallon of diesel fuel to obtain a savings of $\$ 1,580$ per truck per year. On a national scale of approximately 2.2 million heavy vehicles ${ }^{10}$, this translates to $\$ 3.5$ billion per year in fuel cost savings. Given this appreciable amount, it is clearly evident that these second-generation drag reduction devices could have a major impact on the United States' economy if they were to be implemented on a nationwide basis.

\section{Summary and Conclusions}

Through this experimental study, we have investigated several second-generation drag reduction devices. The wind-averaged drag coefficient computed at $65 \mathrm{mph}$ is used as a means of comparing the performance of trailer skirts and base flaps. Of the trailer skirts that are tested, the long wedge skirt provides the greatest drag reduction by combining the performance of the short wedge skirt at small yaw angles with that of the straight side skirts at larger yaw angles. The angled and curved base flap devices yield reductions in the wind-averaged drag coefficient approximately eight to nine times greater than that of the long wedge skirt. For the angled base flaps, the optimum angles of deflection are found to be unequal between the side and top flaps with the top flap having a slightly greater angle of deflection. For the curved base flaps, the optimum radius of curvature is about equal to the width of the trailer. The results of this study are similar to those of Cooper; namely, that the second-generation trailer underbody and base drag reduction devices are capable of significantly reducing the national fuel usage of the trucking 
industry. What is needed to get these devices onto operating heavy vehicles is a committed effort between the government, the tractor/trailer manufacturers, and the trucking fleets. The government has seen the opportunity to improve the fuel economy of heavy vehicles and has taken the initiative to support research and development in heavy vehicle aerodynamics. The involvement of tractor/trailer manufacturers can provide expertise in both road testing and design issues of these devices. The trucking fleets can give practical insight into how these devices impact the operational capability of their fleets and foresee any potential concerns. Only until this collaboration is established will the nation be able to benefit from the significant cost savings that these second-generation drag reduction devices can provide.

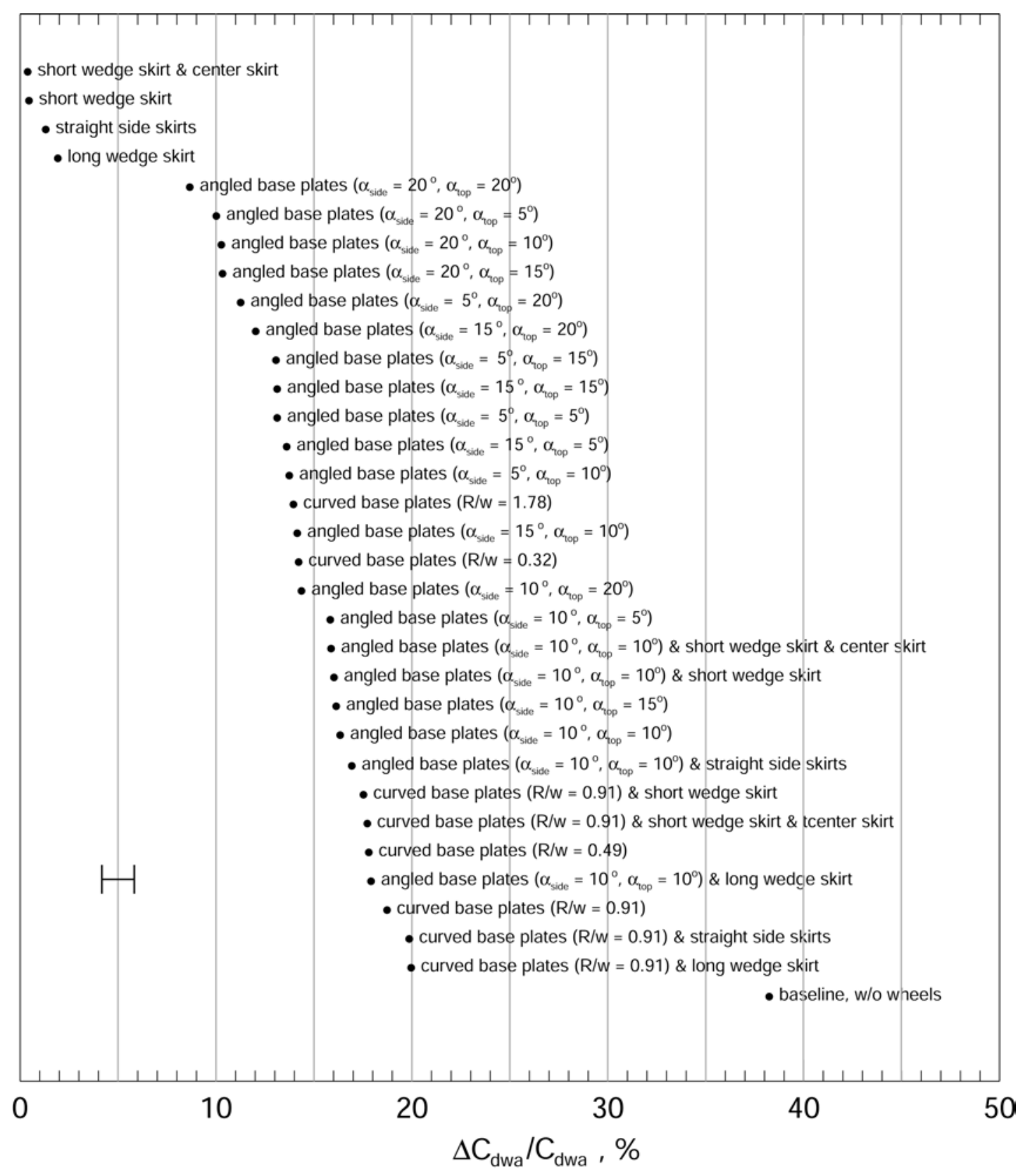

Figure 10. Percent reduction in the wind-averaged drag coefficients relative to the baseline configuration for all of the test cases. 


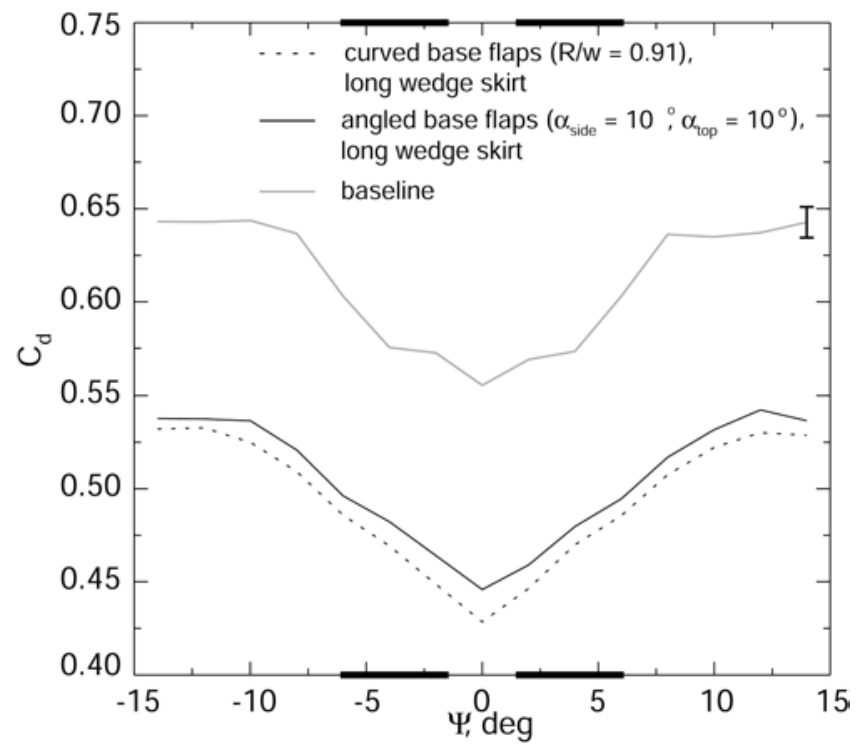

Figure 11. Drag coefficient curves for the model with a combination of the curved base flaps $(R / w=0.91)$ and the long wedge skirt and the model with a combination of the angled base flaps $\left(\alpha_{\text {top }}=10^{\circ}, \alpha_{\text {side }}=10^{\circ}\right)$ and the long wedge skirt.

\begin{tabular}{|l|c|c|}
\hline \multicolumn{1}{|c|}{ Configuration } & $C_{d w a}( \pm 0.004)$ & $\Delta C_{d w a} / C_{d w a}, \%( \pm 0.8 \%)$ \\
\hline Baseline & 0.590 & - \\
\hline Baseline w/o wheels & 0.364 & 38.3 \\
\hline Baseline w/ long wedge skirt & 0.578 & 2.0 \\
\hline Baselinew/ short wedge skirt & 0.587 & 0.1 \\
\hline $\begin{array}{l}\text { Baseline w/ short wedge skirt and } \\
\text { center skirt }\end{array}$ & 0.587 & 0.1 \\
\hline Baseline w/ straight side skirts & 0.582 & 1.4 \\
\hline $\begin{array}{l}\text { Baseline w/ angled base flaps }\left(\alpha_{\text {top }}=\right. \\
\left.10^{\circ}, \alpha_{\text {side }}=10^{\circ}\right)\end{array}$ & 0.493 & 18.4 \\
\hline $\begin{array}{l}\text { Baseline w/ curved base flaps }(R / w= \\
0.91)\end{array}$ & 0.479 & 18.0 \\
\hline $\begin{array}{l}\text { Baseline w/ angled base flaps }\left(\alpha_{\text {top }}=\right. \\
\left.10^{\circ}, \alpha_{\text {side }}=10^{\circ}\right) \text { and long wedge skirt }\end{array}$ & 0.484 & 20.0 \\
\hline $\begin{array}{l}\text { Baseline } \mathrm{w} / \text { curved base flaps }(R / w= \\
0.91) \text { and long wedge skirt }\end{array}$ & 0.472 & \\
\hline
\end{tabular}

Table 1. Wind-averaged drag coefficient, $C_{d w a}$, and the percent reduction in the wind-averaged drag coefficient relative to the baseline case for various model configurations.

\section{Acknowledgments}

This work was funded by the Department of Energy Office of Heavy Vehicles Technologies under the direction of Dr. Sid Diamond. The authors wish to thank Dennis Acosta, Kurt Long, Rabi Mehta, Jim Ross, Dale Satran, Bruce Storms, and Dave Yaste of the NASA Ames Fluid Mechanics Laboratory for their guidance in conducting the wind 
tunnel experiments. This work was performed under the auspices of the U.S. Department of Energy by the University of California, Lawrence Livermore National Laboratory under Contract No. W-7405-Eng-48.

\section{References}

${ }^{1}$ Eberhardt, J.J., "Overview of the DOE Heavy Vehicle Technologies R\&D Program," Paper No. 1999-01-2235, SAE Government/Industry Meeting, Washington, DC, April 26-28, 1999.

${ }^{2}$ Cooper, K.R., "Truck Aerodynamics Reborn-Lessons from the Past,” SAE Paper No. 2003-01-3376, 2003.

3“OTT's Office of Heavy Vehicle Technologies: Program Overview," www.trucks.doe.gov, Office of Transportation Technologies, United States Department of Energy, February 2001.

${ }^{4}$ Cooper, K.R., "The Effect of Front-Edge Rounding and Rear-Edge Shaping on the Aerodynamic Drag of Bluff Vehicles in Ground Proximity," Paper No. 850288, SAE International Congress, Detroit, MI, February 25-March 1, 1985.

${ }^{5}$ Hammache, M., Michaelian, M., and Browand, F., "Aerodynamic Forces on Truck Models, Including Two Trucks in Tandem," Paper No. 2002-01-0530, SAE World Congress, Detroit, MI, March 4-7, 2002.

${ }^{6}$ BDAS 9.2, Software Package, Aerospace Computing, Inc., 2003.

${ }^{7}$ Ingram, K.C., "The Wind-Averaged Drag Coefficient Applied to Heavy Goods Vehicles," Transport and Road Research Laboratory Supplementary Report 392, 1978.

${ }^{8}$ Salari, K. and McWherter-Payne, M., "Computational Flow Modeling of a Simplified Integrated Tractor-Trailer Geometry," Sandia Report SAND2003-3383, 2003.

${ }^{9}$ Storms, B.L., Ross, J.C., Heineck, J.T., Walker, S.M., Driver, D.M., and Zilliac, G.G., "An Experimental Study of the Ground Transportation System (GTS) Model in the NASA Ames 7- by 10-Ft Wind Tunnel," NASA/TM-2001-209621.

${ }^{10}$ Davis, S.C. and Diegel, S.W., "Transportation Energy Data Book: Edition 23," ORNL-6970, Oak Ridge National Laboratory, October 2003. 